\title{
LIGHT CURVE SOLUTIONS OF THE ECCENTRIC BINARIES KIC 10992733, KIC 5632781, KIC 10026136 AND THEIR OUT-OF-ECLIPSE VARIABILITY
}

\author{
Diana Kjurkchieva and Doroteya Vasileva \\ Department of Physics and Astronomy, Shumen University, 115 Universitetska, 9700 \\ Shumen, Bulgaria
}

\begin{abstract}
We determined the orbits and stellar parameters of three eccentric eclipsing binaries by light curve solutions of their Kepler data. KIC 10992733 and KIC 5632781 undergo total eclipses while KIC 10026136 reveals partial eclipses. The components of the targets are G and K stars. KIC 10992733 exhibited variations which were attributed to variable visibility of spot(s) on asynchronously rotating component. KIC 5632781 and KIC 1002613 reveal tidally-induced features at periastron, i.e. they might be considered as eclipsing heartbeat stars. The characteristics of the periastron features (shape, width and amplitude) confirm the theoretical predictions.
\end{abstract}

Key words: binaries: eclipsing - methods: data analysis - stars:

fundamental parameters - stars: individual (KIC 10992733, KIC 5632781, KIC 10026136)

\section{INTRODUCTION}

The eccentric binaries are important objects for the modern astrophysics because they present probes for study of tidal interaction. The gradual loss of energy due to tidal forces leads to circularization of the orbit and the synchronization of the rotation of these stars with their orbital motion (Regos et al. 2005).

Recently we are witnesses of the next stage of development of the tidal interaction theory and testing of its predictions. The unprecedented highaccuracy Kepler observations (Borucki et al. 2010, Koch et al. 2010) allowed 
Table 1: Parameters of the targets from the EB catalog: orbital period $P$, mean temperature $T_{m}$, widths $w_{1,2}$ of the eclipses (in phase units), depths of the eclipses $d_{1,2}$ (in flux units), phase of the secondary eclipse $\varphi_{2}$

\begin{tabular}{cccccccc}
\hline \hline Kepler ID & $P[\mathrm{~d}]$ & $T_{m}[\mathrm{~K}]$ & $w_{1}$ & $w_{2}$ & $d_{1}$ & $d_{2}$ & $\varphi_{2}$ \\
\hline 10992733 & 18.525 & 5274 & 0.0113 & 0.0153 & 0.405 & 0.268 & 0.72 \\
5632781 & 11.025 & 5786 & 0.04 & 0.0457 & 0.395 & 0.376 & 0.66 \\
10026136 & 9.08 & 6292 & 0.0361 & 0.0532 & 0.3124 & 0.2529 & 0.65 \\
\hline \hline
\end{tabular}

to discover and investigate new, fine, tidally-induced effects predicted by Kumar et al. (1995): light feature at the periastron and tidally-excited oscillations (Welsh et al. 2011, Fuller \& Lai 2011, Burkart et al. 2012, Thompson et al. 2012, Nicholls \& Wood 2012, Hambleton et al. 2013a). The newly discovered objects were called "heartbeat" (HB) stars. They provide important tests for the stellar astrophysics and information about the stellar interiors (Hambleton et al. 2013b).

The number of HB stars found in the Kepler data gradually increases (Kirk et al. 2016). Some of them have been objects of follow-up spectroscopy which shows a good agreement between the spectroscopic and photometric orbital elements (Smullen \& Kobulnicky 2015, Shporer et al. 2016, Dimitrov et al. 2017). Last years we also found 12 HB stars between several samples of eclipsing Kepler systems (Kjurkchieva \& Vasileva 2015, 2016, Kjurkchieva et al. 2016a, b).

The goal of this study is determination of the orbits and physical parameters of three eccentric binaries, KIC 10992733, KIC 5632781 and KIC 10026136, as well as searching for tidally-excited effects. Table 1 presents available information for the targets from the EB catalog (Prsa et al. 2011, Slawson et al. 2011, Kirk et al. 2016).

\section{Light curve solutions}

The modeling of Kepler data (Archive MAST) was carried out by the package PHOEBE (Prsa \& Zwitter 2005). We used its mode "Detached binaries" because our targets are detached systems, which is clearly true from their light curves (Fig. 1).

Long cadence (LC) data from many quarters are available in the Kepler archive for these binaries. We worked with the live version of the Kepler EB catalog (http://keplerebs.villanova.edu/). To ignore the effect of accidental light fluctuations in the procedure of the light curve solutions and to obtain 

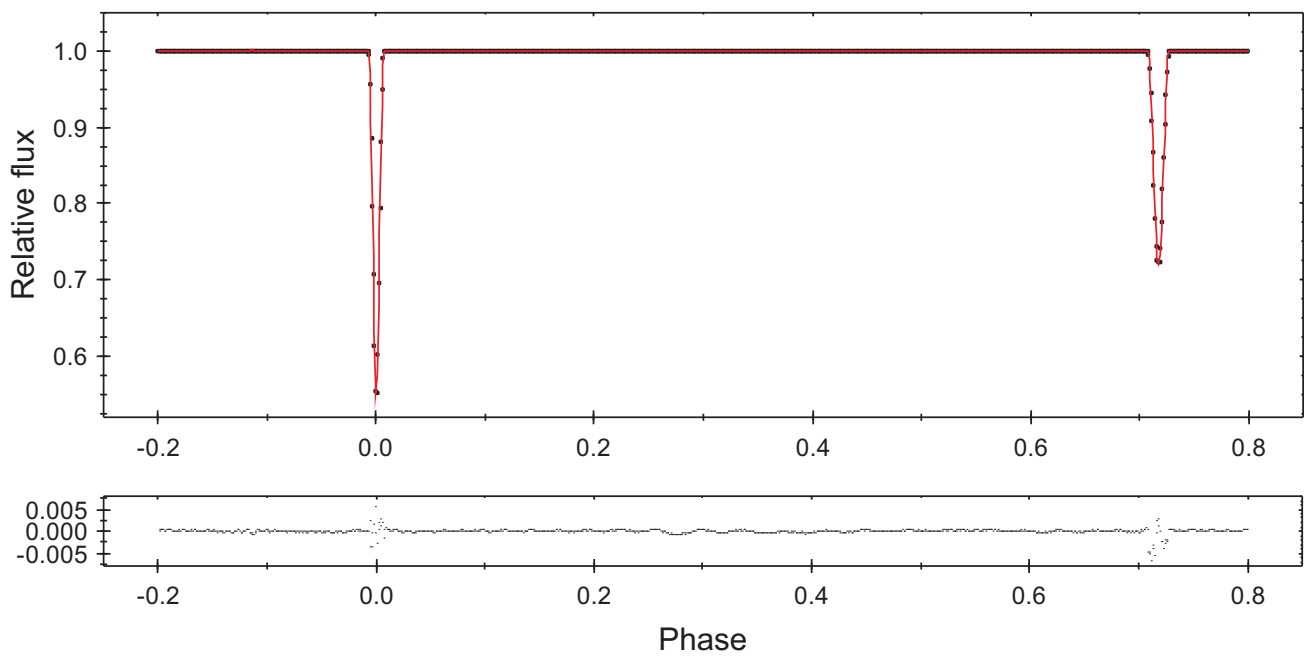

Figure 1: Top panel: the binned folded Kepler data of KIC 10992733 (black points) with their PHOEBE fit (red line); Bottom panel: the corresponding residuals from the model subtracted data for all phases (including eclipses)

adequate configuration parameters we modelled all available LC photometric data (above 50000 points for each target) after appropriate phase binning. For this aim we applied the PHOEBE option for binning of data. We used 1000 bins in phase (the smaller value leads to loss of information from the eclipses).

The modeling procedure is described in Kjurkchieva \& Vasileva (2015). Shortly it consists of several steps: fixing $T_{1}$ to target temperature $T_{m}$; preliminary calculation of the eccentricity $e$ and periastron angle $\omega$; varying of $e$ and $\omega$ to fit the eclipse phases; varying of the inclination $i$, mass ratio $q$, secondary temperature $T_{2}$ and potentials $\Omega_{1,2}$ to reproduce the whole curve; adjusting the real stellar temperatures $T_{1}^{f}$ and $T_{2}^{f}$ around the target value $T_{m}$ by the formulae (Ivanov et al. 2010):

$$
\begin{gathered}
T_{1}^{f}=T_{m}+s \frac{\Delta T}{s+1}, \\
T_{2}^{f}=T_{1}-\Delta T,
\end{gathered}
$$

where $s=l_{2} / l_{1}$ (ratio of relative luminosities) and $\Delta T=T_{m}-T_{2}$ were taken from the last PHOEBE fitting. 

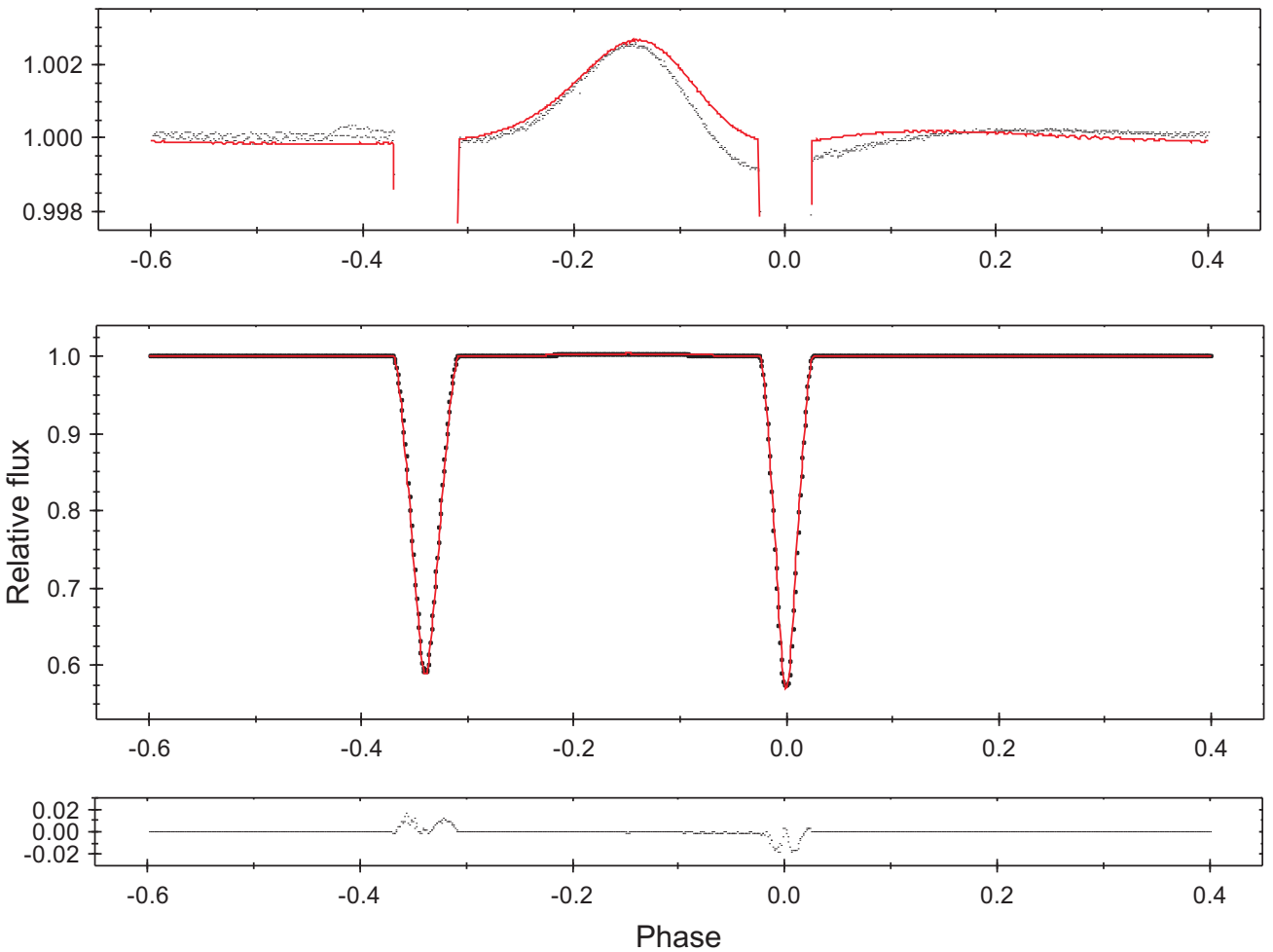

Figure 2: Central panel: the binned folded Kepler data of KIC 5632781 (black points) with their PHOEBE fit (red line); Bottom panel: the corresponding residuals from the model subtracted data for all phases (including eclipses); Top panel: magnified view of the out-of-eclipse flux variation 

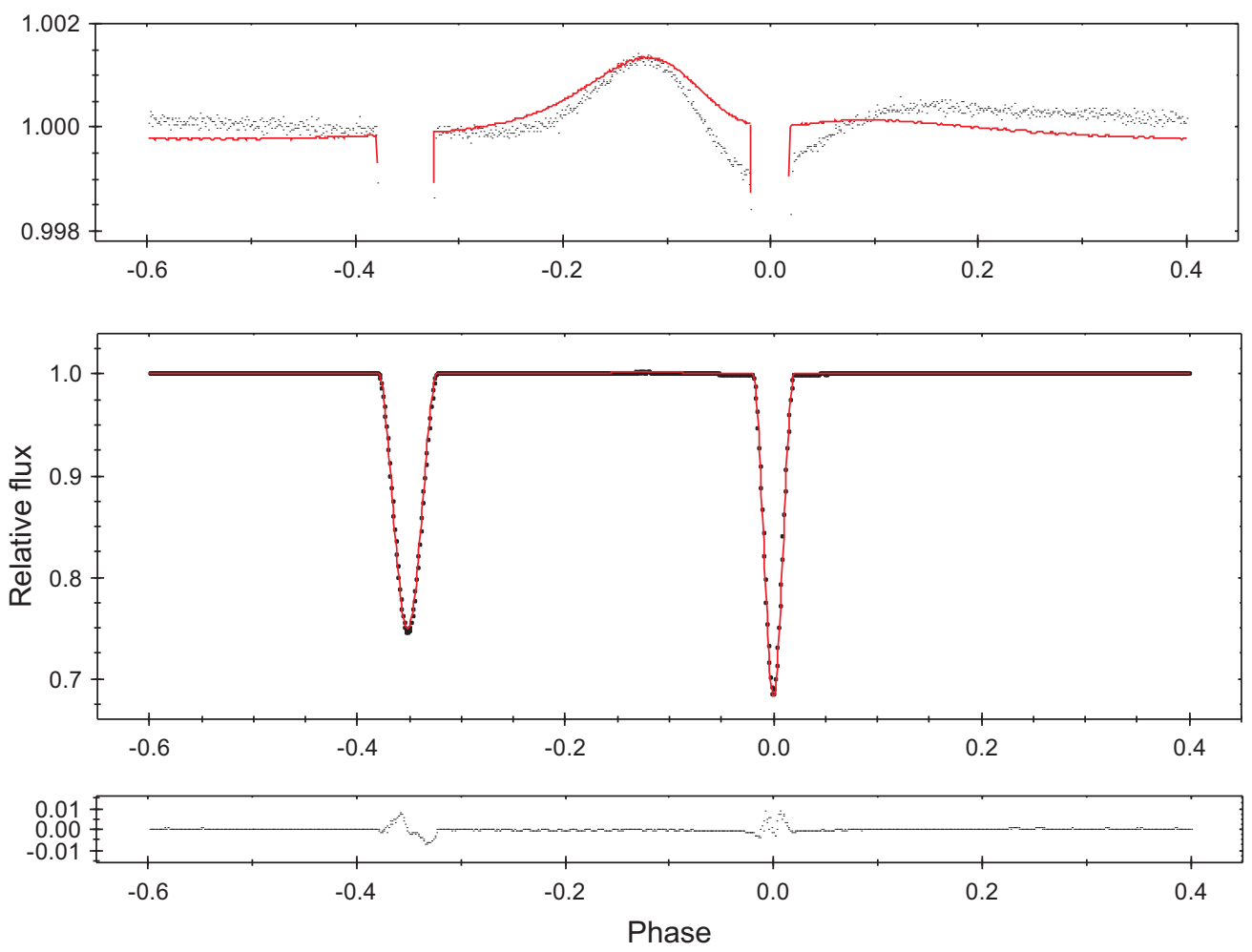

Figure 3: The same as figure 2 for KIC 10026136

Table 2: Fitted and calculated parameters of the light curve solutions: eccentricity $e$; periastron angle $\omega$; inclination $i$; mass ratio $q$; temperature of the secondary star $T_{2}$; surface potentials $\Omega_{1,2}$; relative radius of the primary and secondary stars $r_{1,2}$; periastron phase $\varphi_{\text {per }}$; ratio of the relative luminosities of the stellar components $l_{2} / l_{1}$; calculated temperatures of the primary and secondary stars $T_{1,2}^{f}$

\begin{tabular}{cccc}
\hline \hline Kepler ID & 10992733 & 5632781 & 10026136 \\
\hline$e$ & $0.380(1)$ & $0.273(2)$ & $0.296(1)$ \\
$\omega[\mathrm{deg}]$ & $25.34(1)$ & $22.5(1)$ & $35.95(1)$ \\
$i[\mathrm{deg}]$ & $89.92(6)$ & $89.2(2)$ & $87.33(2)$ \\
$q$ & $0.509(3)$ & $0.97(1)$ & $0.42(1)$ \\
$T_{2}[\mathrm{~K}]$ & $4890(24)$ & $5739(20)$ & $6193(20)$ \\
$\Omega_{1}$ & $32.8(2)$ & $11.41(4)$ & $11.1(2)$ \\
$\Omega_{2}$ & $23.3(1)$ & $13.01(5)$ & $8.7(1)$ \\
\hline$r_{1}$ & $0.031(1)$ & $0.0993(4)$ & $0.094(1)$ \\
$r_{2}$ & $0.023(1)$ & $0.0834(4)$ & $0.061(1)$ \\
$\varphi_{p e r}$ & 0.915 & 0.886 & 0.921 \\
$l_{2} / l_{1}$ & 0.471 & 0.685 & 0.392 \\
$T_{1}[\mathrm{~K}]$ & $5386(35)$ & $5805(16)$ & $6319(14)$ \\
$T_{2}[\mathrm{~K}]$ & $4995(23)$ & $5758(20)$ & $6220(20)$ \\
\hline \hline
\end{tabular}


Although PHOEBE works with potentials, it gives a possibility to calculate directly all values (polar, point, side, and back) of the relative radius $r_{i}=R_{i} / a$ of each component ( $R_{i}$ is linear radius and $a$ is orbital separation). Moreover, PHOEBE yields as output parameters bolometric magnitudes $M_{b o l}^{i}$ of the two components in conditional units (when radial velocity data are not available). But their difference $M_{b o l}^{2}-M_{b o l}^{1}$ determines the true luminosity ratio $s=L_{2} / L_{1}=l_{2} / l_{1}$.

The method of differential corrections was used as a fitting algorithm. The parameters of the best light curve solutions are given in Table 2 while the corresponding synthetic curves are shown in Figs. 1-3 as continuous lines. The residual curves show bigger scatters during the eclipse phases (Figs. 13). Similar behavior could be seen also for other Kepler binaries (Hambleton et al. 2013a, Hambleton et al. 2013b, Lehmann et al. 2013, Maceroni et al. 2014). It was attributed to the effects of finite integration time (Kipping 2010). The reasons for this effect may be also numerical imperfectness of the physical model (Prsa et al. 2016) as well as contribution of pulsations and spots.

The formal PHOEBE errors of the fitted parameters were unreasonably small. That is why we estimated the parameter errors manually based on the following rule (Dimitrov et al. 2017). The error of parameter $b$ corresponded to that deviation $\Delta b$ from its final value $b^{f}$ for which the mean residuals of the HB features increase by $3 \bar{\sigma}$ ( $\bar{\sigma}$ is the mean photometric error of the target).

The light curve solutions show that: (i) KIC 10992733 and KIC 5632781 undergo total eclipses and their mass ratios should be considered with a big confidence (Terrell \& Wilson 2005); (ii) the targets have moderate eccentricity (0.27-0.38); (iii) the temperatures of target components are close (within $400 \mathrm{~K}$, Table 2); (iv) mass ratios are in the range 0.42-0.97.

\section{Out-of-eclipse variability of the targets}

The out-of-eclipse light of KIC 10992733 contains semi-regular oscillations (Fig. 4) with periods 5-7 d which undergo long-term modulation with amplitudes up to 0.005 mag. Their shape as well as lack of strong periodicity seem to exclude pulsation explanation. This is confirmed by the periodogram analysis of the out-of-eclipse data that reveals bad-defined peak (Fig. 5). Its period $P_{\text {out }}=5.137$ days is not harmonic of the orbital period $(18.525 \mathrm{~d})$. The ratio $P_{\text {orb }} / P_{\text {out }} \sim 3.6$ of KIC 10992733 differs almost twice from the ratio 


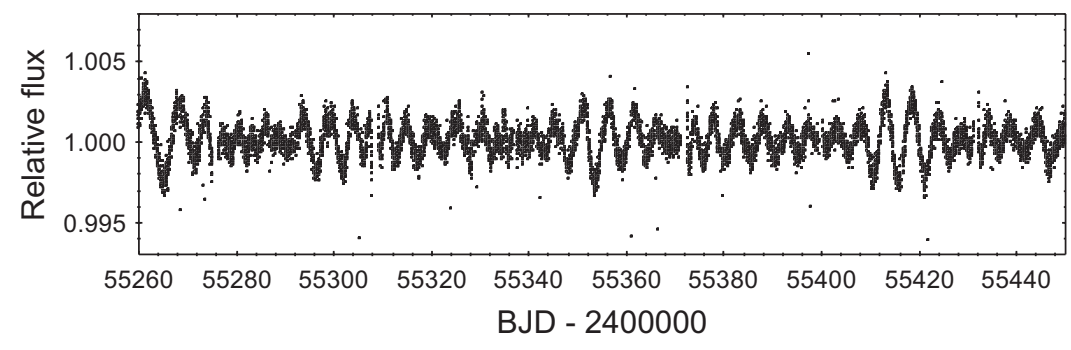

Figure 4: The small-amplitude oscillations of KIC 10992733 probably are due to photospheric spot.

of the pseudo-synchronous angular velocity and mean motion $\Omega_{p s} / n \sim 1.96$ for $e=0.38$ (Hut 1981). Probably, $P_{\text {out }}=5.137$ days presents rotational period of some of the target components. Then the observed out-of-eclipse variability may be explained by variable spot visibility, the bad-defined peak of the Fourier transform may be result of differential rotation while the amplitude modulation may be attributed to spot activity cycle. The components of KIC 10992733 are K stars for which it is inherent characteristic. The asynchronism of the binary is expected considering its high eccentricity.

Cool spot with angular size of order of $10^{\circ}$ may reproduce the observed amplitude of out-of-eclipse oscillations of KIC 10992733. But there is a problem with the shape of oscillations: if the system is coplanar one spot of highly-inclined configuration would cause light variation whose shape is flat during almost half a cycle, different from the observed one. This problem may be overcame if one supposes that KIC 10992733 is not coplanar binary (expected for asynchronous system), i.e. far away from equilibrium state. Another alternative is KIC 10992733 to be coplanar binary with two diametrically opposed spots on some of its components but with a period of $2 P_{\text {out }}=10.274 \mathrm{~d}$. Then the ratio $P_{\text {orb }} / 2 P_{\text {out }} \sim 1.8$ almost corresponds to pseudo-synchronous state of KIC 10992733. Precise spectral observations in the future may solve this ambiguity.

The out-of-eclipse light curves of KIC 5632781 and KIC 10026136 reveal light features around the periastron phases (Figs. 2-3, top panels) with amplitudes of $0.0035 \mathrm{mag}$ and $0.0025 \mathrm{mag}$, i.e. these targets are eclipsing heartbeat stars (EB+HB type, Dimitrov et al. 2017). None of them reveals tidally-induced pulsations (Figs. 2-4).

The synthetic PHOEBE features at the periastron reproduce well (but 


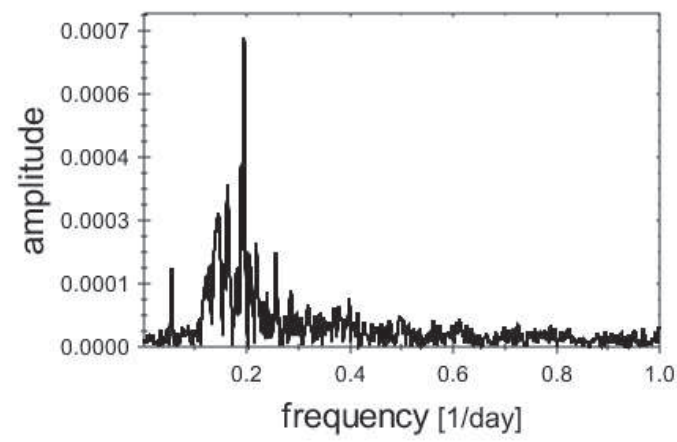

Figure 5: Periodogram of out-of-eclipse variations of KIC 10992733

not perfectly) the observed ones. The possibility to reproduce such mmag details is a compliment of the physical model of binary stars, particularly of PHOEBE.

The mean temperatures of KIC 5632781 and KIC 10026136 (Table 1) correspond to the maximum of temperature distribution of the MS heartbeat stars (figure 2 in Hambleton et al. 2013b).

According to the model of Kumar et al. (1995) the amplitude of the periastron feature depends on the separation of the objects and their masses while its shape depends on the orbit parameters (Hambleton et al. 2013b): the argument of periastron $\omega$ determines the symmetry, the eccentricity $e$ determines its relative width and the inclination $i$ dictates the peak to trough ratio of the tidal pulse (see fig. 5 of Thompson et al. 2012). The contributions of the different parameters on the shape, width and amplitude of the periastron feature are apparent in figs. 3-4 of Dimitrov et al. (2017). It should be pointed out that both model sets (Thompson et al. 2012 and Dimitrov et al. 2017) describe result of clean tidal distortions without contribution of reflection and other faint effects. The last ones may be considerable for some cases (as KOI-54).

The tidally-induced periastron features of KIC 5632781 and KIC 10026136 confirm the theoretical predictions.

(a) Their widths (around $0.40-0.45$ in phase units) correspond to eccentricities of around 0.3 .

(b) The tidally-induced periastron features have a "hook" shape consisting of two parts with almost the same widths: light increasing followed by light trough (that coincides with the primary eclipse). This shape is ex- 
pected for systems with $0 \leq \omega \leq 90^{\circ}$ (see fig. 3 of Dimitrov et al. 2017). The arguments of periastron $\omega$ of our two HB targets (Table 2) fulfil this condition.

(c) The ratios between the amplitudes of the two sections of the periastron features, the hump-shape part and the trough-shape part, of our targets are almost 1:1 (Figs. 2-3). This corresponds to binaries with $\omega \sim 30^{\circ}$ (fig. 3 of Dimitrov et al. 2017).

The noneclipsing stars KIC 3547874, KIC 7622059 and KIC 11494130 from the sample of Thompson et al. (2012) exhibit tidally-induced hookshape periastron features similar to ours. But their amplitudes of 1690, 1020 and $600 \mathrm{ppm}$ are considerably smaller than those of our two targets. This result may be explained by almost 2 times shorter periods of our KIC 5632781 and KIC 10026136 than those of KIC 3547874, KIC 7622059 and KIC 11494130, because the shorter period means smaller separation, the stronger tidal interaction and correspondingly the bigger amplitude of the tidally-induced periastron feature.

\section{Conclusions}

This paper presents the results of determination of the orbits and parameters of stellar configurations of the eclipsing eccentric binaries KIC 10992733, KIC 5632781 and KIC 10026136 on the basis of their Kepler data. The outof-eclipse light variations of KIC 10992733 probably due to spot(s). KIC 5632781 and KIC 10026136 are eclipsing HB stars. The characteristics of their tidally-induced features are consistent with the theoretical predictions.

The presented study adds new two members to the family of HB binaries and provides new data to search for dependencies of the tidally-induced effects of eccentric binaries on their orbital and stellar parameters.

\section{Acknowledgments}

This work was supported partly by project DN08/20 of the Fund for Scientific Research of the Bulgarian Ministry of Education and Science and by project RD 08-102/03.02.17 of Shumen University. It used the SIMBAD database and NASA Astrophysics Data System Abstract Service. We used data from Kepler EB catalog (http://keplerebs.villanova.edu/). The authors are very grateful to the anonymous referee for the valuable recommendations and notes. 


\section{References}

Borucki W. et al., 2010, Sci, 327, 977

Burkart, J., Quataert, E., Arras, P., Weinberg, N.N., 2012, MNRAS, 421, 983

Claret, A., Gimenez, A., 1993, A \& A, 277, 487

Dimitrov D., Kjurkchieva D., Iliev I., 2017, MNRAS, 469, 2089

Fuller, J., Lai, D., 2011, MNRAS, 420, 3126

Hambleton, K. et al., 2013a, MNRAS, 434, 925

Hambleton, K., Degroote, P., Conroy, K., et al. 2013b, EAS Publications Series, 64, 285 1, 4.6

Hut, P., 1981, A \& A, 99, 126

Ivanov V.P., Kjurkchieva D.P., Srinivasa Rao M., 2010, BASI, 38, 83

Kipping, M., 2010, MNRAS, 408, 1758

Kirk, B. et al., 2016, AJ, 151, 68

Kjurkchieva, D., Vasileva, D., 2015, PASA, 32, 23

Kjurkchieva, D., Vasileva, D., 2016, NewA, 48, 30

Kjurkchieva, D., Vasileva, D., Dimitrov, D., 2016a, ApSS, 361, 132

Kjurkchieva, D., Vasileva, D., Dimitrov, D., 2016b, AJ, 152. 189

Koch, D.G. et al., 2010, ApJ, 713, L79

Kopal, Z., 1978, ASSL, 68, Dordrecht, D. Reidel Publishing Co.

Kumar, P., Ao, C., Quataert, E., 1995, ApJ 449, 294

Lajoie, C., Sills, A., 2011, ApJ, 726, 67

Lehmann, H. et al., 2013, A \& A, 557A, 79

Maceroni, C. et al., 2014, A \& A, 563A, 59 
Nicholls, C.P., Wood, P.R., 2012, MNRAS, 421, 2616

Prsa, A., Zwitter, T., 2005, ApJ, 628, 426

Prsa, A. et al., 2011, AJ, 141, 83

Regos, E., Bailey, V., Mardling, R., 2005, MNRAS, 358, 544

Sepinsky, J.F. et al., 2007, ApJ, 660, 1624

Sepinsky, J.F. et al., 2009, ApJ, 702, 1387

Slawson, R. et al., 2011, AJ, 142, 160

Smullen, R., Kobulnicky, H., 2015, ApJ, 808, 166S

Shporer A. et al., 2016, ApJ, 829, 34

Terrell D., Wilson R., 2005, ApSpSci, 296, 221

Thompson, S.E. et al., 2012, ApJ, 753, 86

Welsh, W. et al., 2011, ApJS, 197, 4

Willems, B., Claret, A., 2005, SPC, 333, 52 